\title{
Factores comportamentales para enfermedades no transmisibles en estudiantes Universitarios.
}

\author{
Behavioral factors for non-communicable diseases in University students \\ Fatores comportamentais para doenças não transmissíveis em estudantes universitários \\ Andrés Felipe Villaquiran-Hurtado* \\ Sandra Jimena Jácome-Velasco ** \\ Erica Benavides-Ortega ${ }^{* * *}$
}

\begin{abstract}
Autor de correspondencia
* Fisioterapeuta. Magister en Intervención integral en el deportista. Docente Universidad del Cauca. Correo: avillaquiran@ unicauca.edu.co. (D) https://orcid.org/00000002-6156-6425. Popayán, Colombia.

** Fisioterapeuta. Magister en Ciencias de la Educación. Docente Universidad del Cauca. Correo: sjacome@unicauca.edu.co, (ID) https://orcid.org/0000-0001-6605-8377. Popayán, Colombia.

*** Estudiante de X semestre del Programa de Fisioterapia. Universidad del Cauca. Correo: erbenavides@unicauca.edu.co. (D) https:// orcid.org/0000-0003-3576-8346. Popayán, Colombia.
\end{abstract}

\section{Resumen}

Introducción: La universidad trae consigo nuevos retos para los jóvenes estudiantes; los estilos de vida que se adopten durante esta etapa, serán importantes para mantener una calidad de vida adecuada o deteriorar su salud con la aparición de enfermedades no transmisibles. Objetivos: Determinar los factores comportamentales relacionados con las enfermedades no transmisibles en los estudiantes de la facultad de Ciencias de la Salud y Ciencias Agrarias de una universidad pública del Cauca. Materiales y Métodos: Estudio descriptivo y observacional realizado a 202 estudiantes seleccionados mediante muestreo aleatorio simple, quienes diligenciaron el cuestionario básico y ampliado del método progresivo de la OMS para la vigilancia de los factores de riesgo de las enfermedades crónicas (STEPS) validado por la OMS; se evaluaron los pasos 1 y 2 del cuestionario y cada uno de los pasos cuenta con 6 y 3 ítems respectivamente. Resultados: El cáncer es el principal antecedente familiar con el 51,5\%; el 55,4 \% de los estudiantes ha pasado sin comer al menos una de las 3 comidas esenciales en la última semana; solo el $51 \%$ realiza actividad física. Una gran mayoría de universitarios ( $73,8 \%$ ) consume bebidas alcohólicas, con un aumento en el consumo de un 26,7 \% al ingresar a la Universidad, también el 17,3 \% fuma. Conclusiones: Los estudiantes universitarios presentaron comportamientos poco saludables que los ponen en un mayor riesgo de sufrir Enfermedades No Transmisibles.

Palabras Clave: Enfermedad crónica, factores de riesgo, hábitos, estilo de vida.
Este es un artículo bajo la licencia CC BY (https://creativecommons.org/ licenses/by/4.0/) @) (1)

\section{Abstract}

Introduction: University brings new challenges for young students; the lifestyle students adopt in this stage will be important to maintain an adequate life quality or deteriorate their health with the emergence of non-communicable diseases. Objectives: Determine behavioral factors related to non-communicable diseases for students of the Health Sciences and Agricultural Sciences Faculties, in a public university in Cauca. 
Materials and Methods: Descriptive and observational study performed on 202 students selected through a simple aleatory sample, who filled both the basic and comprehensive questionnaires of the WHO STEPwise approach to Surveillance (STEPS) method, to monitor the risk factors of chronic diseases; the steps 1 and 2 of the questionnaire were evaluated, each of the steps is constituted by 6 and 3 items, respectively. Results: Cancer is the main family precedent (51.5\%); 55.4\% of the students have spent the last week without eating at least one of the 3 essential meals of the day; only $51 \%$ exercise. Most of the university students (73.85) drink alcoholic beverages, with a $26.7 \%$ increase of consumption when admitted to the university, also $17.3 \%$ smoke. Conclusions: Students show unhealthy behaviors that put them at a higher risk of contracting non-communicable diseases.

Keywords: Chronic disease, risk factors, habits, lifestyle.

\section{Resumo}

Introdução: A universidade traz consigo novos desafios para os jovens estudantes; os estilos de vida que se adotem durante esta etapa, serão importantes para manter uma qualidade de vida adequada ou deteriorar sua saúde com a aparição de doenças não transmissíveis. Objetivos: Determinar os fatores comportamentais relacionados com as doenças não transmissíveis nos estudantes da Faculdade de Ciências da Saúde e Ciências Agrarias de uma universidade pública do estado Cauca, Colômbia. Materiais e Métodos: Estudo descritivo e observacional realizado a 202 estudantes selecionados através da amostragem aleatória simples, os quais diligenciaram o questionário básico e ampliado do método progressivo da OMS para a vigilância dos fatores de risco das doenças crónicas (STEPS) validado pela OMS; se avaliaram os passos 1 e 2 do questionário e cada um dos passos conta com 6 e 3 itens respectivamente. Resultados: O câncer é o principal antecedente familiar com o 51,5\%; o 55,4 \% dos estudantes tem passado sem comer ao menos uma das 3 refeições essenciais na última semana; só o $51 \%$ realiza atividade física. Uma grande maioria de universitários $(73,8 \%)$ consume bebidas alcoólicas, com um aumento no consumo de um 26,7 \% ao ingressar à Universidade, também o 17,3 \% fuma. Conclusões: Os estudantes universitários apresentam comportamentos pouco saudáveis que os põem num maior risco de sofrer Doenças Não Transmissíveis.

Palavras-chave: Doença crónica, estilo de vida, fatores de risco, hábitos. $\frac{\frac{\text { E-ISSN 2322-7028 }_{\text {Vol. } 15 \text { No. } 2}}{\text { Jul - Dic } 2018}}{\text { Cúcuta, Colombia }}$

\section{Introducción}

El creciente aumento de las enfermedades no transmisibles (ENT) durante este siglo, según Glasgow (1), ha generado un problema de salud pública mundial, siendo una de las principales causas de morbilidad y mortalidad. Las enfermedades cardiovasculares, la diabetes, el cáncer y las enfermedades respiratorias crónicas reconocidas como ENT, generaron en el 2012 más de 38 millones de muertes en el mundo como fue citado en los artículos de Pulido y la OMS (1-3).

Según Martínez (4) en Colombia entre el 2008 y el 2012 se registraron 727.146 (75\%) muertes asociadas directamente con ENT, con una tasa de mortalidad global en los cinco años de 319,5 fallecidos por 100.000 habitantes y el $4,4 \%$ de estas muertes se produjeron en personas con un nivel educativo superior. Para Llamas (5), la enfermedad cardiovascular es la principal causa de muerte asociada a los estilos de vida. De acuerdo con los datos publicados por la Secretaría Departamental de Salud del Cauca, el reporte ASIS de Morbilidad específica por sexo y edad por Municipio, para PopayánCauca en el año 2015, en población joven se presentó una morbilidad de 49 casos de diabetes, 338 de enfermedades cardiovasculares, 7.575 de enfermedad respiratoria, 1.965 de neoplasias y 3.394 de otras neoplasias, sumando un total de 13.321 casos que al dividirlos por 95.718 personas calculadas en las proyecciones del DANE para Popayán en el año 2015, con edades entre los 16 y 35 años, se obtuvo una prevalencia del 13,92\%.

Los estilos de vida considerados como aspectos del comportamiento, la conducta y la rutina cotidiana, dependen de la decisión personal y realizados de forma voluntaria o no, pueden promover la salud o deteriorarla reduciendo la esperanza de vida $(6,7)$. Por otra parte, acciones como consumo de alcohol y tabaco, ingesta de alimentos poco saludables, comportamiento sedentario y poca actividad física son factores comportamentales de riesgo para la aparición de ENT (8) que además de deteriorar la salud, afectan el desempeño académico de los estudiantes pudiendo generar pérdida de asignaturas o alta repitencia como indicador de bajo o mal rendimiento 
ISSN-PRINT

1794-9831

E-ISSN 2322-7028

Vol. 15 No. 2

Jul - Dic 2018

Cúcuta, Colombia académico; lo que significa que existe una estrecha relación entre el rendimiento escolar y el consumo de alcohol y cigarrillo (9).

Algo semejante ocurre con los comportamientos que se acentúan sobre la vida estudiantil universitaria como son: el paso entre la adolescencia y la adultez joven, la exigencia de los estudios, los cambios psicosociales que conlleva la estancia universitaria, que pueden contribuir a la modificación en los horarios de alimentación, inactividad física, estrés, aumento del consumo de bebidas embriagantes y alta prevalencia de sedentarismo $(6,10-12)$.

En este sentido, diferentes investigacioneshan demostrado la problemática y vulnerabilidad que presentan los universitarios $(8,13)$. En una revisión realizada sobre factores de riesgo cardiovascular en estudiantes universitarios se pudo concluir que es un periodo crítico, propenso a enfermedades no transmisibles, debido a un bajo consumo de fruta y verdura, alto consumo de grasas, sobrepeso y obesidad, pre hipertensión arterial y alto consumo de cigarrillo (10).

El objetivo de este estudio es determinar los factores comportamentales que influyen en el desarrollo de enfermedades no transmisibles (ENT) en estudiantes de las facultades de Ciencias de la Salud y Ciencias Agrarias de la Universidad del Cauca, con el fin de evidenciar la problemática de salud y en futuros proyectos proponer mejores entornos saludables para el estudiante, a fin de promover una formación más integral.

Los aportes de este estudio, además de permitir generar una línea de base para hábitos y estilos de vida saludables, facilitarán a las directivas institucionalesla toma de decisiones sobre los programas de bienestar estudiantil a desarrollar en un futuro próximo. Así mismo, permitirán a la población conocer los factores de riesgo, contribuyendo a la toma de conciencia y de decisiones en el empoderamiento de sus procesos en salud. Es importante considerar para una población la disminución de los factores de riesgo sobre enfermedades crónicas, para que en un futuro sea más saludable y productiva, lo cual se verá reflejado en la economía y calidad de vida de la región y el país.

\section{Objetivos}

\section{Objetivo General}

Conocer los factores comportamentales relacionados con las enfermedades no transmisibles en los estudiantes de las facultades de Ciencias Agrarias y Ciencias de la salud de una Universidad pública del Cauca.

\section{Objetivos específicos}

- Determinar las características sociodemográficas y biomédicas de los estudiantes de la Universidad del Cauca.

- Caracterizar los aspectos académicos de los estudiantes de la Universidad.

- Identificar los factores comportamentales sobre enfermedades no transmisibles de los estudiantes universitarios.

- Establecer posibles relaciones entre características sociodemográficas y repitencia académica con respecto a los factores comportamentales sobre enfermedades no transmisibles de los estudiantes universitarios.

\section{Materiales y Métodos}

Estudio observacional, descriptivo de corte transversal, realizado en estudiantes de una Universidad pública del Departamento del Cauca matriculados en el primer periodo académico de 2016.

Para calcular el tamaño de la muestra se utilizó el programa EPIDAT versión 3.1, con un tamaño de población de (2.728) estudiantes de la Facultad de Ciencias de la Salud y Ciencias Agrarias, con una prevalencia de $13,92 \%$, nivel de confianza de $95 \%$ y precisión de $5 \%$ requiriéndose un mínimo de 174 participantes. Se contó con un total de 202 estudiantes seleccionados mediante muestreo aleatorio simple, quienes cumplieron con los criterios de tipificación: encontrarse activos en el sistema de registro académico del primer periodo académico del año 2016, pertenecer a una de las carreras ofertadas por cada facultad y manifestar su aceptación hacia la participación en el estudio.

A cada participante se le explicó el objetivo de la investigación y la metodología, previo a la firma del consentimiento informado y posterior aplicación de la encuesta. La encuesta de recolección de datos se construyó basada en las mediciones de comportamiento del paso 1 del método progresivo de la OMS para vigilancia de factores de riesgo de las enfermedades crónicas (STEPS) (14), en los items relacionados con: consumo de alcohol, cigarrillo, dieta, actividad física, antecedentes de tensión 
arterial elevada y las medidas físicas; del paso 2 se tuvieron en cuenta: estatura, peso y perímetro de cintura y cadera; se adicionaron preguntas de interés académico. $\mathrm{Al}$ instrumento se le realizó aseguramiento de la validez mediante prueba piloto en el año 2014, con una población equivalente al 14,8\% de la muestra, contando con 30 personas con características similares a la población objeto de estudio. Se encontró que todas las preguntas realizadas fueron comprendidas y respondidas por los participantes en un tiempo promedio de 20 minutos, sin necesidad de modificar el flujo de preguntas.

El instrumento permitió identificar aspectos demográficos, antecedentes en salud (tensión arterial elevada, diabetes), mediciones del comportamiento (actividad física, consumo de tabaco, consumo alcohol, dieta) y medidas físicas (talla, peso, Índice de masa corporal, perímetro de cintura, perímetro de cadera) relacionados con el desarrollo de ENT. En el cuestionario se incluyeron preguntas relacionadas con el ámbito académico (semestre, carrera, promedio de carrera, repetición de asignaturas y ocupación adicional) con el fin de identificar si existía relación entre los factores comportamentales sobre enfermedades no trasmisibles y la repitencia de asignaturas como indicador del mal o bajo rendimiento académico dentro de los aspectos académicos indagados.

Posterior a la encuesta, se procedió a realizar la toma de las medidas antropométricas a cada uno de los participantes; se midió la talla y peso por medio de un tallímetro y báscula mecánica, previamente calibrada con confirmación de exactitud y precisión con pesas de $10 \mathrm{~kg}$, $20 \mathrm{~kg}$ y $30 \mathrm{~kg}$.; se continuó con la toma de los perímetros de la cintura y la cadera, por medio de una cinta métrica de 1.50 metros, calibrada mediante comparación de su valor con la equivalencia proporcionada por un instrumento patrón de medición trazable; las medidas fueron tomadas por una persona capacitada, teniendo como referente las recomendaciones de la International Society for the Advancement of Kinanthropometry (ISAK) (15).

Para el procesamiento de la información se empleó el programa PSPP (alternativa gratuita al programa SPSS) y R wizard, ambos softwares de uso libre bajo licencia GNU. Con respecto a la interpretación de los resultados se realizó un análisis descriptivo con distribuciones de frecuencia y porcentaje; posteriormente se efectuó un análisis inferencial, para lo cual se utilizaron las pruebas no paramétricas U de Mann Whitney y chi cuadrado; luego se realizaron pruebas de correlación, con cálculo del OR. Todas las pruebas se tomaron con un nivel de confianza del $95 \%$.

Para la realización de la investigación se tuvieron en cuenta los aspectos éticos de la declaración de HELSINKI por la Asociación Médica Mundial (AAM); así como la resolución 8430 de 1993 del Ministerio de Salud, que establece las normas científicas y administrativas para la investigación en salud y con seres humanos en Colombia; igualmente, se tuvo en cuenta el aval del Comité de ética de la facultad Ciencias de la Salud de la Universidad $(16,17)$.

\section{Resultados}

Se realizaron 202 encuestas a estudiantes de los programas de Fisioterapia, Enfermería, Medicina y Fonoaudiología de la Facultad de Ciencias de la Salud y a los programas de Ingeniería Forestal, Ingeniería Agroindustrial e Ingeniería Agropecuaria de la Facultad de Ciencias Agrarias durante el primer periodo del año 2016.

El género femenino predominó en el 60,9\% $(n=123)$ de la población, con promedio de edad de 21 años, el 94,6 $\%(\mathrm{n}=191)$ son solteros, el 78,7 \% $(\mathrm{n}=159)$ de estudiantes procede de la zona urbana, el 46,0 \% $(n=93)$ pertenecen régimen subsidiado en salud, el $81,7 \%$ de los estudiantes viven en estratos 1 al 3 y tan solo el 11,9\% estudia y trabaja al mismo tiempo.

En cuanto a las medidas antropométricas el promedio del peso fue de $59,37 \mathrm{~kg}$ y una talla promedio de $160 \mathrm{~cm}$, el 73,3 \% presentó un Índice de Masa Corporal (IMC) catalogado como normo peso, la mayoría de universitarios $(74,3 \%)$ no presentaron riesgo cardiovascular con la evaluación del índice Cintura Cadera (ICC).

Respecto a los antecedentes familiares: el cáncer presenta una frecuencia del 52,5\%, seguido de la diabetes en un $51 \%(\mathrm{n}=103)$, e hipertensión arterial con el 49,5\% $(\mathrm{n}=100)$; de los antecedentes personales se encontró mayor frecuencia de enfermedad respiratoria en el 14,9 $\%(n=30)$, seguida de Hipertensión arterial con un 5,5 $\%(\mathrm{n}=11)$ y problemas de lípidos y colesterol en un porcentaje de 5,5\% $(n=11)$. El 48,5\% de la población encuestada se realiza los chequeos preventivos por lo menos una vez al año ( $\mathrm{n}=98)$, el $24,8 \%$ se efectúa el autoexamen de mama o testículos periódicamente $(n=50)$ y el 31,2 \% de las mujeres se practica la citología anualmente $(\mathrm{n}=63)$.
E-ISSN 2322-7028

Vol. 15 No. 2

Jul - Dic 2018

Cúcuta, Colombia 
ISSN-PRINT

1794-9831

E-ISSN 2322-7028

Vol. 15 No. 2

Jul - Dic 2018

Cúcuta, Colombia
En relación con los aspectos académicos, el $53 \%$ se encuentra cursando del primero al quinto semestre de sus carreras, con un promedio de carrera entre 3,0 y 3,9 en el $62,4 \%$ de los estudiantes y con pérdida de asignaturas en el $52,0 \%$ de los encuestados.

Refiriéndose a los hábitos nutricionales, cabe resaltar que el $81,2 \%$ manifiesta que la alimentación es muy importante, el 46,5\% consume más de tres comidas al día y el $66,3 \%$ manifiesta que casi siempre consume alimentos en horarios fijos. El 57,9 \% consume mayor cantidad de carbohidratos a la semana, con un consumo diario entre 1 a 2 vasos de agua (39,1\%); tan solo el 38,1 $\%$ consume frutas y verduras una vez a la semana. Del mismo modo, el 31,2 \% agrega más sal a los alimentos previamente preparados, el 30,2\% añade más azúcar a los alimentos previamente preparados $(\mathrm{n}=61)$; el $61,4 \%$ de los encuestados consume con frecuencia alimentos ricos en azúcares $(\mathrm{n}=124)$, el 63,9 \% ingiere comidas rápidas 1 a 2 veces por semana $(n=129)$, el 55,4\% ha dejado de comer al menos una de las 3 comidas esenciales la última semana $(n=112)$ debido a falta de tiempo con un 35,6\% $(\mathrm{n}=72)$.

Sobre los hábitos de actividad física y transporte, se encontró que: el medio de desplazamiento más utilizado por la población encuestada es caminar con un $40,1 \%$, el $51,0 \%$ realiza actividad física, el $62,1 \%$ lo hace por salud y bienestar, el $30 \%$ de la población practica el trote y el $36,1 \%$ considera que tiene un estilo de vida sedentario (ver tabla 1).

Tabla 1. Actividad física de los estudiantes universitarios.

\begin{tabular}{|c|c|c|}
\hline Características & $\mathbf{N}$ & $\%$ \\
\hline \multicolumn{3}{|l|}{ Realiza actividad física } \\
\hline $\mathrm{Si}$ & 103 & 51 \\
\hline No & 99 & 49 \\
\hline \multicolumn{3}{|c|}{ Frecuencia semanal para realizar actividad física } \\
\hline Todos los días & 20 & 19,4 \\
\hline Dos o más veces por semana & 68 & 66 \\
\hline Una vez por semana & 12 & 11,6 \\
\hline No lo realiza con regularidad & 3 & 3 \\
\hline \multicolumn{3}{|l|}{ Tiempo empleado para la actividad física } \\
\hline Menos de 30 minutos & 17 & 16,5 \\
\hline Entre 30 minutos y una hora & 41 & 39,8 \\
\hline Entre una y dos horas & 41 & 39,8 \\
\hline Más de dos horas & 4 & 3,8 \\
\hline \multicolumn{3}{|l|}{ Razones para realizar actividad física } \\
\hline Recreación & 33 & 32 \\
\hline Salud y bienestar & 64 & 62,1 \\
\hline Mejorar la apariencia física & 6 & 5,8 \\
\hline \multicolumn{3}{|l|}{ Tipo de actividad realizada } \\
\hline Caminar por más de 30 minutos & 25 & 24,2 \\
\hline Trotar & 31 & 30 \\
\hline Nadar & 13 & 12,6 \\
\hline Montar bicicleta & 27 & 26,2 \\
\hline Otro & 7 & 6,7 \\
\hline \multicolumn{3}{|l|}{ Razones para no realizar actividad física } \\
\hline Falta de tiempo & 60 & 60,6 \\
\hline Mis condiciones físicas no lo permiten & 1 & 1,1 \\
\hline Falta de interés & 33 & 33,3 \\
\hline Otro & 3 & 3,3 \\
\hline No responde & 1 & 1,1 \\
\hline \multicolumn{3}{|c|}{ Practica algún deporte a nivel competitivo } \\
\hline $\mathrm{Si}$ & 27 & 13,4 \\
\hline No & 175 & 88,6 \\
\hline \multicolumn{3}{|l|}{ Tiempo de práctica deportiva } \\
\hline Menos de un año & 3 & 11,1 \\
\hline Entre 1 y 3 años & 12 & 44,4 \\
\hline 3 a 5 años & 1 & 3,7 \\
\hline Más de 5 años & 11 & 40,7 \\
\hline
\end{tabular}

Fuente: Instrumento de recolección de datos 
Respecto a la práctica de hábitos nocivos referidos al consumo de alcohol y cigarrillo, el 73,8 \% dice consumir bebidas alcohólicas y el $69,7 \%$ bebe por diversión, el $26,7 \%$ expresa haber aumentado su consumo al ingresar a estudiar a la Universidad. En cuanto al hábito de fumar: el 17,3\% afirma que lo hace permanentemente, mientras que el $22,8 \%$ de los estudiantes se considera fumador pasivo (Ver tabla 2).
1794-9831

E-ISSN 2322-7028

Vol. 15 No. 2

Jul - Dic 2018

Cúcuta, Colombia

Tabla 2. Características de hábitos nocivos de los estudiantes universitarios

\begin{tabular}{|c|c|c|}
\hline Características & $\mathrm{N}$ & $\%$ \\
\hline \multicolumn{3}{|l|}{ Consumo de alcohol } \\
\hline $\mathrm{Si}$ & 149 & 73,8 \\
\hline No & 53 & 26,2 \\
\hline \multicolumn{3}{|c|}{ Frecuencia Consumo de bebidas embriagantes } \\
\hline 2 a 4 veces a la semana & 3 & 1,5 \\
\hline Todos los días & 1 & 0,5 \\
\hline Todos los fines de semana & 20 & 9,9 \\
\hline $1 \mathrm{vez}$ al mes & 30 & 14,9 \\
\hline Rara vez & 93 & 46 \\
\hline Nunca & 53 & 26,2 \\
\hline No sabe/ no responde & 2 & 1 \\
\hline \multicolumn{3}{|c|}{ Razón por la cual consume bebidas alcohólicas } \\
\hline Diversión & 104 & 69,7 \\
\hline Soledad & 6 & 4 \\
\hline Problemas personales & 2 & 1,3 \\
\hline Carga académica & 6 & 4 \\
\hline Otro & 31 & 20,8 \\
\hline \multicolumn{3}{|c|}{ Consume alcohol hasta embriagarse } \\
\hline $\mathrm{Si}$ & 39 & 19,3 \\
\hline No & 163 & 80,7 \\
\hline \multicolumn{3}{|c|}{ Aumento el consumo de bebidas alcohólicas al ingresar a la Universidad } \\
\hline $\mathrm{Si}$ & 54 & 26,7 \\
\hline No & 148 & 73,3 \\
\hline \multicolumn{3}{|c|}{ Le resulta difícil decir que No cuando lo invitan a tomar bebidas alcohólicas } \\
\hline $\mathrm{Si}$ & 31 & 15,3 \\
\hline No & 171 & 84,7 \\
\hline \multicolumn{3}{|l|}{ Fuma } \\
\hline $\mathrm{Si}$ & 35 & 17,3 \\
\hline No & 167 & 82,7 \\
\hline \multicolumn{3}{|l|}{ Hace cuánto tiempo fuma } \\
\hline Menos de 1 año & 7 & 3,5 \\
\hline Entre 1 y 3 años & 14 & 6,9 \\
\hline De 3 a 5 años & 3 & 1,5 \\
\hline Más de 5 años & 11 & 5,4 \\
\hline Nunca he fumado & 162 & 80,2 \\
\hline No sabe/no responde & 5 & 2,5 \\
\hline \multicolumn{3}{|c|}{ Cuantos cigarrillos fuma al día } \\
\hline Uno & 25 & 71,4 \\
\hline Entre dos y cinco & 7 & 20 \\
\hline Entre seis y diez & 2 & 5,7 \\
\hline Más de diez & 1 & 2,8 \\
\hline \multicolumn{3}{|c|}{ Estaría dispuesto a seguir un programa para dejar de fumar } \\
\hline $\mathrm{Si}$ & 19 & 54 \\
\hline No & 16 & 46 \\
\hline \multicolumn{3}{|c|}{ Se considera fumador pasivo } \\
\hline $\mathrm{Si}$ & 46 & 22,8 \\
\hline No & 135 & 66,8 \\
\hline No sabe/ no responde & 21 & 10,4 \\
\hline
\end{tabular}

Fuente: Instrumento de recolección de datos. 
ISSN-PRINT

$1794-9831$

E-ISSN 2322-7028

Vol. 15 No. 2

Jul - Dic 2018

Cúcuta, Colombia
En el análisis inferencial se encontró que existe relación significativa entre género y sedentarismo ( $\mathrm{p}=0,001 ;$ OR:2,72; IC95\%:1,51-4,9), siendo el género femenino 2,72 veces más sedentario que el masculino; en cuanto al consumo de alcohol y consumo hasta la embriaguez, el género femenino parece ser un factor protector $(\mathrm{p}=0,033 ; \mathrm{OR}: 0,32 ; \mathrm{IC} 95 \%: 0,15-0,36)$. Por otra parte, el consumo de alcohol hasta la embriaguez se muestra como un factor de riesgo para la pérdida de asignaturas ( $\mathrm{p}=0,041$; OR 2,127;IC95\%:1,022$4,427)$, encontrándose un alto número de estudiantes en condición de repitencia de asignaturas con un $51,98 \%(n=105)$ (Tablas 3 y 5$)$.

Tabla 3. Relación entre género y hábitos nocivos.

\begin{tabular}{|c|c|c|c|c|}
\hline GÉNERO & Sedentarismo & consumo de alcohol & $\begin{array}{l}\text { consumo de alcohol } \\
\text { hasta embriagarse }\end{array}$ & $\begin{array}{c}\text { No consumo de alguna de las } 3 \\
\text { comidas esenciales en la última } \\
\text { semana }\end{array}$ \\
\hline \multirow{3}{*}{$\begin{array}{l}\text { femenino } \\
\text { masculino }\end{array}$} & Sí No & Sí No & Sí No & Sí No \\
\hline & 7251 & 8439 & 15108 & 7746 \\
\hline & 2752 & 6514 & 2455 & 3544 \\
\hline $\mathrm{P}$ & 0,001 & 0,033 & 0,002 & 0,014 \\
\hline \multirow{2}{*}{ OR-IC } & 2,72 & 0,46 & 0,32 & 2,10 \\
\hline & $1,51-4,9$ & $0,23-0,93$ & $0,16-0,66$ & $1,18-3,74$ \\
\hline
\end{tabular}

*P: Valor de $\mathrm{p}$

*OR: Razón de oportunidades

*IC: Intervalo de confianza

Fuente: visor de resultados de PSPP y R wizard

También se encontró asociación entre ICC y consumo de cigarrillo, y diferencia estadísticamente significativa entre edad y consumo de alcohol hasta embriagarse; también se observó en la población objeto de estudio que los participantes menores de 20 años tenían menor riesgo de consumir alcohol hasta la embriaguez (OR:0,38; IC95\%:0,15-0,96) frente a los mayores de 20 años (Tablas 4 y 5)

Tabla 4. Relación entre ICC y consumo de cigarrillo

\begin{tabular}{lll}
\hline & \multicolumn{1}{c}{ Consumo de cigarrillo } \\
\hline ICC & $\mathrm{Si}$ & No \\
Con riesgo & 448 & \\
Sin riesgo & 31119 & \\
& $\mathrm{P}=0,033$ \\
\hline
\end{tabular}

*P: Valor de $\mathrm{p}$

*ICC: índice cintura cadera

Fuente: visor de resultados de PSPP y R wizard 
Tabla 5. Relación entre edad y pérdida de asignaturas con el consumo de alcohol hasta embriagarse

\begin{tabular}{ll}
\hline & Consume alcohol hasta embriagarse \\
\hline EDAD & $\mathrm{Si}$ \\
\hline Menor de 20 años & 653 \\
Mayor de 20 años & 33110 \\
\hline P & 0,035 \\
\hline OR & 0,38 \\
IC & $0,15-0,96$ \\
PÉRDIDA DE ASIGNATURAS & \\
\hline Si & 2679 \\
No & 1384 \\
p & 0,041 \\
OR & 2,127 \\
IC & $1,022-4,427$ \\
\hline
\end{tabular}

*P: Valor de $\mathrm{p}$

*OR: Razón de oportunidades

*IC: Intervalo de confianza

Fuente: visor de resultados de PSPP y R wizard.

\section{Discusión}

Estudios realizados en jóvenes universitarios han demostrado la presencia de factores de riesgo asociados con el estilo de vida como: sedentarismo, tabaquismo, consumo de alcohol, malos hábitos alimenticios, sobrepeso, obesidad y estrés; resultados muy similares a los encontrados en el presente estudio con estudiantes universitarios de dos facultades $(4,6,18)$. Los datos anteriores deben influir sobre las autoridades gubernamentales para que prioricen su atención sobre los factores que inciden en la salud de los jóvenes universitarios, puesto que esta problemática tiene raíces en los hábitos aprendidos desde la infancia y es influenciada por otros factores como los conductuales, los ambientales, los socioeconómicos y los genéticos. Factores que se refuerzan en la vida adulta, sobre todo, en el paso por la universidad en donde se incrementa el consumo de alcohol y tabaco, así como la práctica inadecuada de hábitos alimenticios y la disminución de la actividad física como se mencionará más adelante (7).

Dentro de este marco de investigación, se observa que un gran porcentaje de estudiantes caucanos presentan un IMC normal y sin riesgo cardiovascular, resultados muy similares a los encontrados por Ureña et al. (19) en estudiantes de enfermería, aunque lo encontrado difiere con varios estudios (20-22), en donde se demuestra que los parámetros de sobrepeso y de grasa aparecen con porcentajes elevados; al respecto es importante mencionar que la edad juega un papel muy importante en el IMC, sin embargo, se debe decir que esta medida ha sido bastante cuestionada por falta de precisión sobre la composición corporal (porcentaje de grasa, porcentaje muscular, estatura), lo que hace que se tienda a reportar resultados generalizados sin una adecuada interpretación, generando resultados antropométricos erróneos, frente a lo cual, se sugiere para próximos estudios se conside el índice de Rohrer como una alternativa más fiable para la estimación del grado de obesidad (23).

Por otra parte, el incremento progresivo en el número de personas con sobrepeso y obesidad, producto de comportamientos no saludables ha generado una mayor incidencia de diabetes, hipertensión y dislipidemias, siendo más frecuente la aparición de enfermedades cardiovasculares(24). Sobreestaproblemática, el presente estudio, muestra que existen diferencias según género en lo que se refiere a los hábitos de alimentación; al respecto se encontró que las mujeres están más propensas al no consumo de las tres comidas esenciales, a diferencia, de lo reportado en el estudio de Rodríguez, Restrepo y Deossa, quienes encontraron que el género masculino tiene un menor conocimiento sobre aspectos relacionados con la alimentación saludable, lo cual los hace más vulnerables hacia el desarrollo de enfermedades no transmisibles en etapas posteriores (25). En este sentido, por las dinámicas de estudio los universitarios pueden ser más vulnerables 
ISSN-PRINT

1794-9831

E-ISSN 2322-7028

Vol. 15 No. 2

Jul - Dic 2018

Cúcuta, Colombia en comparación con la población en general, debido a la práctica de conductas no saludables (6), las cuales están asociadas con su aparición en edades posteriores, lo cual amerita una continuidad con un estudio de cohorte.

Respecto a los antecedentes familiares, se encontró que el cáncer, la diabetes y la hipertensión son las referencias más comunes entre los universitarios, resultados contrastados con otras investigaciones como la de Cardona y Arroyave (26), quien señala como antecedentes la hipertensión arterial y la enfermedad cerebrovascular, como hechos familiares que preceden a los jóvenes estudiantes. Por otro lado, estos datos también concuerdan con las principales causas de mortalidad en Colombia reseñadas por el ENT entre el 2008 y 2012, presentando 31.600 casos de enfermedades cerebrovasculares en hombres, más de 19 mil casos de diabetes en mujeres y más de 60 mil casos de neoplasias en ambos géneros (4). En consecuencia, estos antecedentes familiares son relevantes sobre la conducta adoptada por los estudiantes, replicándose estas costumbres de generación en generación.

Con referencia, a los hábitos nutricionales de la población relacionados con: el bajo consumo de frutas y agua, la utilización de azúcar y sal en las comidas, la alta ingesta de carbohidratos y alimentos ricos en azucares y el dejar de consumir alguna de las 3 comidas esenciales por falta de tiempo; se debe precisar que son conductas influenciadas por factores psicológicos, fisiológicos y muchas veces por la falta de responsabilidad en el consumo de su propia alimentación $(11,27)$.

También cabe señalar que existe consenso sobre la ingesta inadecuada de alimentación presentada en los universitarios, situación que deben tener en cuenta las instituciones a la hora de planificar y desarrollar los programas de prevención y promoción para favorecer mejores conductas alimentarias (27).

En relación, con la actividad física se sabe que condiciona positivamente la salud del individuo, mejorando la calidad de vida, proporcionándole una mejor condición física, previniendo la mortalidad por ENT. Por otro lado, el nivel de actividad física se ha relacionado positivamente con el rendimiento cognitivo y/o académico (28). Adicionalmente, se ha comprobado que el sedentarismo es uno de los factores de riesgo más frecuente en educandos universitarios (10). En este estudio, cerca de la mitad de los estudiantes no realizaban actividad física aduciendo falta de tiempo, datos que contrastan con lo anteriormente mencionado sobre la inactividad presentada por los estudiantes de educación superior.

Al respecto, estudios como el de Chávez et al. (29), Ayán et al. (30) y el de Mollinedo et al. (31), muestran que el género femenino está más expuesto al sedentarismo que el masculino, siendo esta diferencia significativa en todos los estudios, encontrándose como causantes la falta de tiempo, cansancio y el mismo rol social de la mujer, entre otros. Esto invita a los investigadores a realizar futuros estudios que indaguen sobre esta causalidad en estudiantes de una universidad pública del Cauca, para proponer posibles alternativas de solución frente a esta problemática que afectará a futuro su salud, su bienestar y calidad de vida.

Teniendo en cuenta lo anteriormente expuesto, otro dato aún más preocupante es el consumo de alcohol y el aumento de su ingesta al ingresar a la universidad, actuación que está influenciada por las creencias, la falta de actividad física, la edad, los síntomas depresivos y la falta de conocimientos relacionados con la salud (32); igualmente se le relaciona negativamente con el rendimiento académico (32-33). Llama la atención el consumo de alcohol hasta la embriaguez en estudiantes mayores de 20 años; en relación con este comportamiento Moral etal. (34) afirman que el consumo intenso o abusivo de alcohol disminuye en la medida en que la edad avanza. Sin duda, la ingesta de alcohol tiene complicaciones en la salud individual, y en los problemas de orden familiar y social, debido a la pérdida del control de los impulsos y en algunos casos la exacerbación de conductas violentas e incluso la muerte (35). La asociación entre el consumo de alcohol hasta la embriaguez o también denominado consumo de alcohol abusivo y la pérdida de asignaturas como factor de bajo rendimiento académico, encontrado en el presente estudio, es corroborado por Caballero et al. (36) y por Gómez et al. (9), quienes encontraron esta asociación en poblaciones de estudiantes universitarios.

Por último, el consumo de cigarrillo también puede influir en la aparición de enfermedades crónicas respiratorias, siendo el causante de 6 millones de muertes de personas cada año. De la misma manera como se presenta el consumo de cigarrillo en esta investigación, un estudio realizado por Tami et al. (37) en estudiantes de tercer año de odontología en países latinoamericanos, encontró que un $33 \%$ son fumadores activos, frente a un $45 \%$ de fumadores pasivos en el último mes, cifras que sugieren tomar medidas de prevención y control del uso de cigarrillo (38). 


\section{Conclusiones}

- En el estudio se observó una relación entre la edad y el género, en virtud de que el joven mayor de 20 años se dedica más a la diversión como forma de expresar su preponderante rol masculino; igualmente expresa una tendencia por aumentar el consumo de alcohol; de ahí, que sea importante analizar este fenómeno en futuros estudios.

- Existe un alto número de estudiantes en condición de repitencia de asignaturas, lo cual amerita un estudio a profundidad que permita identificar otros posibles factores asociados a ésta problemática, para poder identificarlos y así establecer sus alternativas de solución

- La falta de actividad física, principalmente en las mujeres por escasez de tiempo, les condiciona negativamente su salud, aumentando la probabilidad de enfermarse en edades de mayor productividad laboral, social y familiar.

- El consumo de carbohidratos, de azúcares, de agregar más sal a los alimentos y de comidas rápidas, sumado al consumo de alcohol, tabaco y falta de actividad física, muestra una población de estudiantes universitarios con tendencia hacia conductas poco asertivas sobre la salud, aumentando la posibilidad de padecer ENT además de afectar su rendimiento académico exponiéndolos a repitencia de asignaturas.

\section{Agradecimientos}

Agradecemos a la Universidad del Cauca y al Programa de Fisioterapia por su colaboración y compromiso con la investigación.

\section{Conflicto de Intereses}

Los autores declaran no tener conflicto de intereses.

\section{Referencias Bibliográficas}

1. WorldHealth Organization. Global statusreport on noncommunicable diseases 2014. [Internet] 2014 [consultado el7octubrede2017];1-302.Disponibleen:http://apps.who.int/iris/bitstream/10665/148114/1/9789241564854 eng.pdf

2. Riley L, Guthold R, Cowan M, Savin S, Bhatti L, et al. The world health organization STEPwise approach to noncommunicable disease risk-factor surveillance: Methods, challenges, and opportunities. Am J Public Health [Internet]. 2016 [consultado el 7 octubre de 2017]; 106(1):74-8. Disponible en: https://www.ncbi.nlm.nih.gov/pmc/articles/PMC4695948/pdf/AJPH.2015.302962.pdf

3. Pulido C, Reyes N, León A, Cárdenas J, Rivera S, Rodríguez A. Factores de riesgo de enfermedades crónicas no transmisibles en estudiantes de ciencias básicas de la escuela de medicina de la universidad pedagógica y tecnológica de Colombia. Rev salud hist sanid [Internet]. 2015 [consultado el 7 octubre de 2017]; 10(1):15-25. Disponible en: http://revistas.uptc.edu.co/index.php/shs/article/view/3989

4. Martínez J. Factores asociados a la mortalidad por enfermedades no transmisibles en Colombia, 20082012. Biomédica [Internet]. 2016 [consultado el 7 octubre de 2017]; 36(4):535-46. Disponible en: http://www.revistabiomedica.org/index.php/biomedica/article/view/3069

5. Llamas Jiménez A. Promoción de hábitos saludables en el trabajo para el bienestar de la población trabajadora. Rev Colomb Cardiol [Internet]. 2014 [consultado el 7 octubre de 2017]; 21(4):199-201. Disponible en: http://dx.doi.org/10.1016/j.rccar.2014.09.001

6. Bastías E, Stiepovich J. Una revisión de los estilos de vida de estudiantes universitarios iberoamericanos. Cienc y enfermería [Internet]. 2014 [consultado el 7 octubre de 2017]; 20(2):93-101. Disponible en: http:// www.scielo.cl/scielo.php?script=sci arttext\&pid=S0717-95532014000200010\&lng=en\&nrm=iso\&tlng=en

7. Londoño D, García J. Prevalencia de factores de riesgo cerebro-cardiovasculares en estudiantes de salud, Armenia- Quindío 2014. Rev Invest Univ Quindío [Internet]. 2015 [consultado el 4 febrero de 2018]; 27(2):91-101. Disponible en: http://www.uniquindio.edu.co/descargar.php?idFile=22519

8. Al Sejari M. Sociocultural Characteristic, Lifestyle, and Metabolic Risk Factors Among a Sample of 
ISSN-PRINT

1794-9831

E-ISSN 2322-7028

Vol. 15 No. 2

Jul - Dic 2018

Cúcuta, Colombia
Kuwaiti Male University Students. Am J Mens Health [Internet]. 2017[consultado el 5 octubre de 2017]; 11(2):308-17. Disponible en: http://journals.sagepub.com/doi/pdf/10.1177/15579888316680937

9. Gómez Cantillo A, Gil Villar Y, Aranzalez Machado V. Consumo de cigarrillo, ingesta de alcohol y su asociación con la percepción de bajo rendimiento académico en estudiantes de ingeniería de una universidad pública. Duazary [Internet]. 2013 [consultado el 1 de may 1 de 2018]; 8(2):169-174. Disponible en: http://revistas.unimagdalena.edu.co/index.php/duazary/article/view/215

10. Morales I, Del Valle R, Soto V, Ivanovic M. Factores de riesgo cardiovascular en estudiantes universitarios. RevChilNutr[Internet].2013 [consultadoel 1octubrede2017];40(4):391-396.Disponible en: https://scielo.conicyt.cl/scielo.php?script=sci arttext\&pid=S0717-75182013000400010

11. Hernández A, Singh P, Andino C, Ulloa C, Daneri A, Flores Z. Caracterización de hábitos relacionados con enfermedades crónicas en población universitaria de Honduras. Rev Cuba Salud Pública [Internet]. 2015 [consultado el 7 octubre de 2017]; 41(2):324-334. Disponible en: http://www.scielosp.org/scielo. php?script $=$ sci arttext\&pid $=$ S0864-34662015000200011\&lang $=$ pt

12. Ramírez Vélez R, Ojeda M, Tordecilla M, Peña J, Meneses J. El consumo regular de bebidas azucaradas incrementa el perfil lipídico-metabólico y los niveles de adiposidad en universitarios de Colombia. Rev Colomb Cardiol [Internet]. 2015 [consultado el 7 octubre de 2017]; 23(1):11-8. Disponible en: https://www.sciencedirect.com/science/article/pii/S0120563315000959

13. Vargas M, Barragán O, Peralta S, Hernández E, Ontiveros M, Favela M. Factores De Riesgo De Enfermedades No Transmisibles En Académicos Universitarios. Rev Ciencias Biológicas y la Salud [Internet]. 2015 [consultado el 7 octubre de 2017]; 18:3-8. Disponible en: https://biotecnia.unison.mx/ index.php/biotecnia/article/view/221

14. Organización Mundial de la Salud. Versión Panamericana del Instrumento STEPS (principal y ampliada). 2010. Disponible en: http://www.who.int/ncds/surveillance/steps/instrument/PAHO STEPS Instrument v2.1 ES.pdf

15. Stewart A, Marfell Jones $M$, Olds $T$, De Ridder H. Protocolo Internacional para la Valoración Antropométrica ISAK. 2011;1-126.

16. República de Colombia. Ministerio de Salud. Resolución $N^{\circ} 008430$ de 1993, octubre 4, por la cual se establecen las normas científicas, técnicas y administrativas para la investigación en salud. [Internet]. Santa Fé de Bogotá: El Ministerio; 1993. [consultado 4 de septiembre de 2014]. Disponible en: https:// www.minsalud.gov.co/Normatividad_Nuevo/RESOLUCION\%208430\%20DE\%201993.pdf

17. Di Ruggiero M. Declaración de Helsinki, principios y valores bioéticos en juego en la investigación Médica con seres humanos. Revista Colombiana de Bioética. [Internet]. 2011 [consultado el 4 de septiembre de 2017]; 6(1):125-144. Disponible en: http://www.redalyc.org/pdf/1892/189219032009.pdf

18. Laguado E, Gomez M. Estilos de vida saludable en estudiantes de enfermería en la universidad cooperativa de Colombia. Rev Hacia la Promoción de la Salud [Internet]. 2014 [consultado el 4 de febrero de 2018];19(1):68-83. Disponible en: http://www.redalyc.org/articulo.oa?id=309131703006

19. Ureña Molina MP, Pacheco Milian M, Rondon Ortega MJ. Conductas alimentarias de riesgo y su relación con la imagen corporal en estudiantes de enfermería. Rev. cienc. cuidad. [Internet]. 2016 [consultado el 14 de noviembre de 2017]; 12(2):57-71. Disponible en: http://revistas.ufps.edu.co/ojs/ index.php/cienciaycuidado/article/view/509

20. Cardozo LA, Cuervo YA, Murcia JA. Porcentaje de grasa corporal y prevalencia de sobrepeso obesidad en estudiantes universitarios de rendimiento deportivo de Bogotá, Colombia. Nutr Clínica y Dietética Hosp [Internet]. 2016 [consultado el 4 de septiembre de 2017]; 36(3):68-75. Disponible en: http://revista.nutricion.org/revista.asp?id=38

21. Mollinedo Montaņo FE, Ortiz Trejo PM, Araujo Espino R, Lugo Balderas LG. Índice De Masa Corporal, Frecuencia Y Motivos de Estudiantes Universitarios Para Realizar Actividad Física. Rev Cuba Educ Medica Super [Internet]. 2013 [consultado el 4 de septiembre de 2017]; 27(3):189-99. 
Disponible en: http://scielo.sld.cu/scielo.php?script=sci arttext\&pid=S0864-21412013000300004

22. González Zapata L, Carreño Aguirre A, Estrada A, Monsalve Álvarez J, Álvarez L. Exceso de peso corporal en estudiantes universitarios según variables sociodemográficas y estilos de vida. Rev Chil Nutr [Internet]. 2017 [consultado el 7 de octubre de 2017]; 44(3):251-261. Disponible en: https:// scielo.conicyt.cl/scielo.php?pid $=\mathrm{S} 0717-75182017000300251 \&$ script $=$ sci arttext

23. Fernández JA, Remesar $X$, Alemany $M$. Ventajas teóricas del índice de Rohrer $\left(\mathrm{P} / \mathrm{A}^{3}\right)$ sobre el índice de masa corporal $\left(\mathrm{P} / \mathrm{A}^{2}\right)$ para estimación de la adiposidad en humanos. Revista Española de Obesidad [Internet]. 2005 [consultado el 15 de abril de 2018]; 3(1):47-55. Disponible en: https://www. researchgate.net/publication/236588100

24. Córdova Villalobos J. La obesidad: la verdadera pandemia del siglo XXI. Cir Cir [Internet]. 2016 [consultado el 7 de octubre de 2017]; 84(5):351-5. Disponible en: http://dx.doi.org/10.1016/j.circir.2016.08.001

25. Rodríguez Espinosa H, Restrepo Betancur L, Deossa G. Conocimientos y prácticas sobre alimentación, salud y ejercicio en universitarios de Medellín- Colombia. Rev Perspect en Nutr Hum [Internet]. 2015 [consultado el 8 de febrero de 2018]; 17(1):36-54. Disponible en: http://aprendeenlinea.udea.edu.co/ revistas/index.php/nutricion/article/view/25434

26. Cardona Arias J, Arroyave Martínez E. Prevalencia de hipertensión arterial en Universitarios, Medellín. Curare [Internet]. 2014 [consultado el 8 de febrero de 2018]; 1(1):17-26. Disponible en: https://revistas.ucc.edu.co/index.php/cu/article/viewFile/304/593

27. Cervera Burriel F, Serrano Urrea R, Vico García C, Milla Tobarra M, García Meseguer MJ. Hábitos alimentarios y evaluación nutricional en una población universitaria. Nutr Hosp [Internet]. 2013 [consultado el 9 de octubre de 2017]; 28(2):438-446. Disponible en: http://www.redalyc.org/ pdf/3092/309227306023.pdf

28. Conde $\mathrm{M}$, Tercedor $\mathrm{P}$. La actividad física, la educación física y la condición física pueden estar relacionadas con el rendimiento académico y cognitivo en jóvenes. Revisión sistemática. Arch Med Deporte [Internet]. 2015 [consultado el 7 de octubre de 2017]; 32(2):100-109.Disponible en: http:// archivosdemedicinadeldeporte.com/articulos/upload/166 rev02.pdf

29. Chávez M, Salazar C, Hoyos G, BautistaA, González D, Ogarrio C. Actividad física y enfermedades crónicas no transmisibles de estudiantes mexicanos en función del género. Retos [Internet]. 2018 [consultado el 6 de febrero de 2018]; 33:169-174. Disponible en: https://recyt.fecyt.es/index.php/retos/article/view/55354

30. Ayán C, Molina A, Varela V, Cancela J, Barrio J, Sánchez V. Relación entre el consumo de tabaco y la práctica deportiva en una población universitaria de ciencias de la salud y la educación. Enfermería Clínica [Internet]. 2017 [consultado el 6 de febrero de 2018]; 27(1):21-7. Disponible en: https://www. sciencedirect.com/science/article/pii/S1130862116301243

31. Mollinedo F, Trejo P, Araujo R, Lugo L. Índice de masa corporal, frecuencia y motivos de estudiantes universitarios para realizar actividad física. Rev Cuba Educ Medica Super [Internet]. 2013 [consultado el 6 de febrero de 2018]; 27(3):189-99. Disponible en: http://scielo.sld.cu/pdf/ems/v27n3/ems04313.pdf

32. Siyan Y, Ngin C, Peltzer K, Pengpid S. Health and behavioral factors associated with binge drinking among university students in nine ASEAN countries. Subst Abuse Treat Prev Policy [Internet]. 2017 [consultado el 7 de octubre de 2017]; 12(1):32. Disponible en: http://substanceabusepolicy. biomedcentral.com/articles/10.1186/s13011-017-0117-2

33. Mekonen T, Fekadu W, Mekonnen TC, Workie SB. Substance Use as a Strong Predictor of Poor Academic Achievement among University Students. Psychiatry J [Internet]. 2017 [consultado el 7 de octubre de 2017]; 2017:1-9. Disponible en: https://www.hindawi.com/journals/psychiatry/2017/7517450/

34. Moral M, Bringas C, Ovejero A, Morales L, Rodríguez F. Emergencia sociosanitaria en consumo de riesgo de alcohol y síntomas de dependencia en Jóvenes. Heal Addict / Salud Y Drog [Internet]. 2017 [consultado el 6 de febrero de 2018]; 17(2):91-99. Disponible en: http://ojs.haaj.org/index.php/haaj/ article/view/307 
35. Tembo C, Burns' S, Kalembo F. The association between levels of alcohol consumption and mental health problems and academic performance among young university students. PLoS One [Internet]. 2017 [consultado el 7 de octubre de 2017]; 12(6):1-13. Disponible en: http://journals.plos.org/plosone/ article?id=10.1371/journal.pone. 0178142

36. Carballo JL, Marín Vila M, Jáuregui Andújar V, García Sánchez, G, Espada, JP, Piqueras, JA. Consumo excesivo de alcohol y rendimiento cognitivo en estudiantes de secundaria de la provincia de Alicante. Salud y drogas [Internet] 2013. [consultado el 8 de febrero de 2018]; 13(2):157-163. Disponible en: http://www.redalyc.org/articulo.oa?id $=83929573008$

37. Tamí Maury I, Silva Vetri MG, Marcano Caldera M, Baasch A, Prokhorov AV. Smoking behavior among third year dental students in Latin American countries: prevalence, perceptions, and risk factors. Salud Publica Mex [Internet]. 2017 [consultado el 7 de octubre de 2017]; 59(2):1-9. Disponible en: https://www.ncbi.nlm.nih.gov/labs/journals/salud-publica-mex/

38. Saridi M, Nanou A, Vasilopoulos C, Kourakos M, Skliros E, Toska A, et al. Smoking habits among greek university students after the financial crisis. Asian Pacific J Cancer Prev [Internet]. 2017 [consultado el 7 de octubre de 2017]; 18(5):1329-35. Disponible en: https://www.ncbi.nlm.nih.gov/ pmc/articles/PMC5555543/ 